\title{
Domestic Applications for Social Robots - an online survey on the influence of appearance and capabilities
}

\author{
Manja Lohse, Frank Hegel, and Britta Wrede
}

\begin{abstract}
Can you imagine a useful task you would like a social robot to perform for you? This paper presents an internet survey where participants were asked this question to identify applications for social robots. The applications mentioned by the participants are based on the appearance of four social robots (AIBO, iCat, BIRON, and BARTHOC) and the information they received about their basic capabilities. It was found that AIBO and iCat seem to be suitable for domestic applications whereas suggested applications for the more functional mobile robot BIRON are situated also in public environments. The anthropomorphic robot BARTHOC mainly seems to be appropriate for public usage. The paper tries to explain how the appearance and the capabilities of the robot influence what applications are ascribed to them. Moreover, it is shown what role domestic robots play in the field of social robotics and how they relate to public robots.
\end{abstract}

Index Terms - robot applications, domestic robots.

\section{INTRODUCTION}

$\mathrm{T}$ HE GENERAL objective of the social robotics research is to design robots that engage in social scenarios which are compelling and familiar to humans. Thus, the robots have to provide a social communicative functionality that is natural and intuitive. This can be supported if appearance and functionality fit the robots' tasks [1] and robots are as selfexplaining as possible. Keeping all this in mind is especially important when designing robots to interact with naïve users in domestic environments.

Today, mainly simple domestic toy robots (e.g. Lego Mindstorms [2], Ugobe's Pleo [3], WowWee's Robosapien [4]) and floor cleaning robots (e.g. Roomba [5]) are sold offthe-shelf. Also some public applications have been developed (e.g. receptionist robot [6], museum guide robots [7], [8]). All these applications have mainly been invented by researchers. They demonstrate the technical functionalities available so far. However, we argue that potential users should be included in the process of finding and developing new scenarios. Altogether, there is a lack of application scenarios and systematic classifications. Researchers do not know what tasks robots should be able to complete for potential users although knowing consumers' opinions is important for designing useful

Manja Lohse, Frank Hegel, and Britta Wrede are with the Applied Computer Science Group, Bielefeld University, Germany.

E-mail: mlohse; fhegel; bwrede@techfak.uni-bielefeld.de applications. Moreover, no guidelines how robots should look like to be suitable for a certain task exist.

In the work presented here this lack of systematic research on robot applications is addressed. The paper introduces an internet survey with potential users that were asked to suggest relevant applications for the four robots $A I B O$ (Sony), iCat (Philips) (both consumer products), BIRON (Bielefeld University) and BARTHOC (Bielefeld University) (both research robots). It is evaluated which of the robots - due to their appearance and functionalities - are rated as being suitable for domestic applications and which applications participants would use themselves.

Section II gives an overview of research on social robots, applications and the importance of the appearance of robots. Section III describes the four robots chosen for the survey based on their appearance. Section IV introduces the method stressing why we decided to conduct an online study. The results of the survey and a discussion are presented in Sections $\mathrm{V}$ to VIII. Finally, in Section IX we conclude what we learned from the study.

\section{RELATED WORK}

Since robots have started to move around our physical and social spaces, their role and our dealings with them have changed significantly. Therefore, a whole research area called social robotics has developed. Social robots are physical entities embodied in a complex, dynamic, and social environment, sufficiently empowered to behave in a manner conducive to their own goals and those of their community [9]. They especially serve as an interface between man and machine [10]. So far, many social robots have been built mainly to demonstrate technical skills within very specific scenarios (e.g. [11]). Even though such robots enable huge advances in research, they are far from being off-the-shelf consumer products. Yet, despite the current situation social robots should become everyday life applications in order to take advantage of their ability to communicate with almost everybody.

When building social robots it has to be strived for a balance between the expectations people have, the capabilities of the machine, and as a result the way to communicate the robot to do specific tasks. Thus, a social robot should be able to communicate with us, understand and even relate to us, in a personal way. It should be capable to understand humans and 
itself in social terms. In turn, human beings should be able to understand the robot in the same social terms, to relate to it and to empathize with it [12].

Bartneck and Forlizzi [13] have created a guideline, which outlines the components a social robot should comprise: First, the form should match the expectations of a user. Second, the robot should communicate verbally and nonverbally with all available modalities. And third, the robot has to be able to take human social norms into consideration.

One domain in which these capabilities are especially useful is domestic applications. In fact, these become increasingly important [14], [15]. "Domestic" describes the place of usage. This place is the home of the user. It is special since the user decides who enters it and creates the rules that apply within it. The robot should be able to perform its specific tasks within this limited domain. Another challenge for domestic applications is that the robot has to learn new tasks within its environment. Learning poses a special problem as the typical user is naïve in programming and unaware of mechanical and control issues. Therefore, new learning methods will have to be developed. Probably the most intuitive and appropriate way to instruct the domestic robots is to interact with it as naturally as humans do in their daily life which again requires robots that, in fact, are social.

\section{A. Applications of Domestic and Social Robots}

Up to now, there are only few commercial applications for both social and domestic robots (see Section I). Developing useful applications for such robots seems to be a challenging task.

Christensen [16] postulates three potential commercial application categories for domestic robots: Entertainment, Everyday Tasks, and Assistance to elderly and handicapped people. These can be differentiated by what he calls a performance metric which specifies requirements for the applications. The performance metric of entertainment applications is forgiving, i.e. the robot is not really required to perform specific tasks - as long as the robot performs interesting interaction sequences the users will in general be satisfied. The challenge for this kind of applications is to provide an open-ended repertoire of interesting interactions to guarantee that the robot does not become boring for the user. In contrast, the performance metric of everyday home tasks for domestic robots is well defined while the individual environment where they will have to fulfil these tasks cannot be anticipated. According to Christensen [16], typical examples for such applications are vacuum cleaning, fetchand-carry tasks, ironing clothes, window cleaning etc. Finally, the category of assistance applications for elderly and handicapped people is mainly motivated by the demographic profiles of occidental societies. The performance metric of these applications is a high degree of flexibility and an easy instruction how to interact with the robots.

Another approach to classify the field has been presented by Fong et al. [17]. In a first survey of socially interactive robotics the authors have mentioned several application fields: social robots as test subjects for research on communication and human development theory, as short-term and long-term service assistants in public and private life, as toys and entertainment devices, for therapy, for research on anthropomorphism, and last but not least in the field of education. These application fields are based on the applications that existed when the paper was written.

Also Ljungblad [18] reported on a workshop held with the goal to conceive potential applications. As a result three interesting scenarios were selected: self-organizing robot plants, robots as travel companions, and amusement park guide robots. These scenarios are rather based on a brainstorming than on a scientific approach to the topic.

Kaplan chose a different approach [19]. By asking himself what would actually make social robots valuable as everyday objects, he came to the conclusion that the value of the objects has to meet the needs of the users. Thus, the robot has to find its place in human life by adding value to it in terms of shortor long-term usage. Based on this hypothesis concrete applications still have to be derived.

Obviously, so far, no single exhaustive categorization of applications for social robots exists even though the question what the systems can be used for has been addressed from different viewpoints. However, it is noticeable that most of these approaches have domestic applications in mind.

\section{B. Appearance of Robots}

Appearance has a major influence on the assumptions people have about applications and functionalities, i.e. behaviours of robots [1]. Current research states that the appearance has to support the correct estimation of the robot's real competencies by the user. The better the user's estimation the less will she be disappointed during the interaction with the robot [19].

In this context, the embodiment of a robot plays a major role. Bartneck [20] has found a facilitation effect in his study with the emotional robot eMuu. Participants acquired a higher score in a negotiation game and they put more effort into the negotiation when they interacted with the embodied robot character instead of the screen character. This may be due to the feeling of social presence [21].

Among very few approaches, Fong et al. [17] define four broad categories of social robots with respect to their appearance: anthropomorphic, zoomorphic, caricatured, and functionally designed robots. An anthropomorphic appearance is recommended to support a meaningful interaction with users [10], [12] because many aspects of nonverbal communication are only understandable if expressed in similarity to a humanlike body. Zoomorphic robots are intended to look like their animal counterparts to support the idea that an observer expects the robot to behave like an animal. In some cases this might be helpful to communicate the functional limitations of a robot. For example, a dog is partly able to understand aspects of human speech but makes many mistakes. This mirrors the 
quality of speech recognition software [20]. Robots with a caricatured appearance are mainly designed both to not elicit any expectations based on familiarity and to focus on very specific attributes like mouth or eyes. Finally, functional robots are designed in a technical/functional manner to illustrate their ultimate functions. This corresponds to the famous claim by Sullivan [22] that form ever follows function. Hence, the designer expects that the user is able to understand the capabilities of the functionally designed robot by looking at its features. For instance, a camera mediates the feature of 'seeing'.

Especially the design of a robot's head is an important issue within human-robot interaction (HRI), because it has been shown that most non-verbal cues are mediated through the face [23]. Without a face the robot is perceived as being anonymous [24]. The physiognomy of a robot changes the perception of its human-likeness, knowledge, and sociability. Therefore, people avoid robots behaving or looking negatively and prefer to interact with positive robots [25]. Furthermore, an expressive face indicating attention [26] and imitating the face of a user [27] makes a robot more compelling to interact with. Consequently, Duffy [9] argues that a robot has to have a certain degree of human-like attributes for meaningful social interaction.

A certain degree of human-like attributes also belongs to the three aspects DiSalvo et al. [28] propose regarding the appearance which should be taken into account when designing robot heads: the robot should have a certain amount of robot-ness to stress the robot's machine capabilities and avoid false expectations of its emotional capabilities; it should have an amount of human-ness to make the user feel comfortable; and finally it should have a certain amount of product-ness that the robot is seen as an appliance.

These aspects are consistent with the matching-hypothesis [1]. This hypothesis claims that appearance and social behaviour of a robot should match the seriousness of the task, because a robot that confirms the expectations people have increases the sense of the robot's compatibility with its task. Especially within the field of domestic applications this seems to be an important aspect in the design of satisfying interactions with naïve users.

Another essential aspect concerning robot design is in what sense users expect the robot to behave like a human and when they attribute human-like qualities to the machine. This phenomenon, which is called anthropomorphism, is in the focus of the following sub-section.

\section{Anthropomorphism and the Uncanny Valley}

It has been shown that the more human-like the appearance of a robot is the more people attribute intentions to the robot within a classical Prison Dilemma Game task [29]. This automatic attribution of human-like qualities is called anthropomorphism. More general, anthropomorphism entails attributing human-like properties, characteristics, or mental states to real or imagined non-human agents and objects [30].
According to v. Foerster [31] humans anthropomorphize because it allows them to explain things they do not understand in terms that they do understand, and what they understand best is themselves as human beings. This is consistent with the familiarity thesis [32] which claims that humans understand the world based upon the mental model of it that they are most familiar with.

According to Guthrie [32], the best explanation for this phenomenon is the best-bet-thesis which is a cognitive and game-theoretic approach to explain anthropomorphism. He claims that humans anthropomorphize because in the face of chronic uncertainty about the nature of the world, guessing that things and events are human-like or have a human cause constitutes a good bet, i.e. if we are right we gain much, but if we are wrong we usually lose little.

Another theoretical approach to the topic is the ThreeFactor-Theory of Anthropomorphism by Epley et al. [30]. They claim that the extent to which people anthropomorphize is mainly determined by one cognitive and two motivational factors: (a) Elicited Agent Knowledge: Knowledge about humans in general or self-knowledge serve as a basis for induction primarily because such knowledge is acquired earlier and is more richly detailed than knowledge about non-human agents or objects. The more human-like in appearance or motion the non-human agent is the more people are likely to use themselves as a source of induction. For example, robots are anthropomorphized more readily when given human-like faces and bodies [28] and hummingbirds suddenly appear more deliberate and thoughtful when their natural quickness is slowed to a human-like speed [33]. The second motivational factor is (b) Effectance Motivation: Effectance describes the need to interact effectively with one's environment. Attributing human characteristics and motivations to non-human agents increases the ability to make sense of an agent's action and reduces uncertainty. Finally, (c) the Sociality Motivation describes the principal need and desire to establish social connections with humans. When people feel lack of social connection they anthropomorphize to a higher extent to satisfy their motivation to be together with others.

Up to now, the phenomenon of human-likeness seems to be the most considered aspect regarding the theory of anthropomorphism in the field of robotics. The hypothesis from anthropomorphism is that the more a robot resembles a human being in appearance the more people expect it to have human-like qualities [9]. Therefore, human-likeness is a special challenge to reach for some researchers (e.g. [34],[35]). Furthermore, the development of robots that closely resemble human beings can contribute to research on cognition [36].

One theory closely connected to anthropomorphism is the Uncanny Valley [37]. It represents how an object can be perceived as having enough human-like characteristics to evoke a constrained degree of empathy through one's ability to rationalize its actions and appearance. When the movements and the appearance are almost human-like but not entirely, there are too many expectations of the capabilities. 
When these are not met the reaction of the observer is most certainly negative. In the end, the object becomes so humanlike that it is effectively treated as a human being where it has re-established a balance between anticipated and actual function and appearance to a sufficient degree that works well [9].

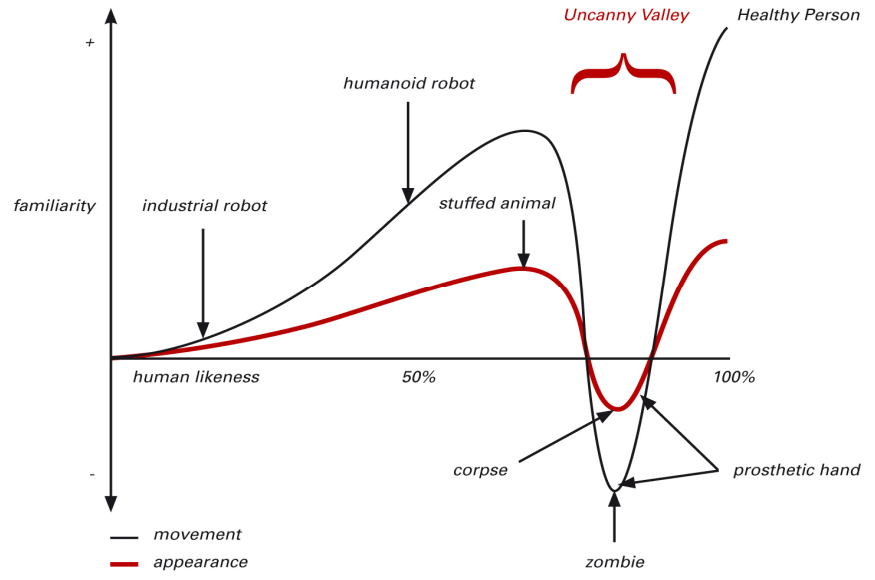

Fig. 1 Uncanny Valley

There are several studies whether the Uncanny Valley exists or not and how to consider the hypothesis when designing human-like robots. For example, [38] was able to find an Uncanny Valley within a study using morphings from a machine-like robot to an anthropomorphic robot. In a further psychological study based on four experiments [39] verified the Uncanny Valley caused by the human visual system. They found that in daily life people rarely confuse artificial faces with real human faces - people do not ask a mannequin in a store for directions to a train station. This suggests that the human visual system has a specific sensitivity to the degree of human-likeness. Therefore the visual system is highly sensitive to abnormalities. In the study it has further been shown that a high degree of abnormality elicits unpleasantness within the tested subjects only if entities exhibit a high degree of realism. For example, an increased eye size within an artificial character did not elicit any unpleasantness, but the same eye size within a human face was sensed as abnormal and therewith unpleasant. Thus, improving the degree of realism of robots without removing abnormal features may lead to an exaggeration of the observers' unpleasant impressions of the artificial faces.

No matter whether the Uncanny Valley exists or not, the theory may serve as a design guideline to not disappoint the expectations of the user. Also it is assumed that within the study presented here the discussed aspects of the robot's appearance have an effect on the applications people derived by seeing short videos of four different robots.

\section{DESCRIPTION OF RobOT SySTEMS}

Since the appearance and functionalities of the robot are in the centre of the survey presented here, this section gives a technical overview of the robot platforms displayed. For each robot the applications and scenarios intended by the developers are mentioned.

\section{A. AIBO (Sony)}

The AIBO Robot ERS-7 is presented in Fig. 4. The design of AIBO is dog-like. The robot uses its four feet to move in its environment. With the acceleration sensors on-board it is able to balance its body. AIBO has - considering its feet, head, ears, and tail - altogether 20 joints (degrees of freedom) which enable the robot to perform dog-like moves.

AIBO has a set of sensors on the head, the back, the chin, and the paws which allow the robot to examine itself and its environment. With the help of the sensors the robot can sense touch. It can perceive sound using a pair of stereo microphones. Therefore, it can react to voice. By means of the colour camera and distance sensors AIBO can recognize colours, faces, and obstacles. It is able to communicate via sounds, a face display, and speech. On the website [39] Sony talks about the "AIBO Entertainment Robot" or even the "World's most popular entertainment robot". Obviously this is the main scenario the developers had in mind.

Moreover, the robot became well known due to the RoboCup league. The Sony website [39] provides more information about the robot.

\section{B. iCat (Philips)}

The iCat shown in Fig. 5 is a plug \& play desktop userinterface robot developed by Philips Research (Eindhoven, the Netherlands). It does not have an on-board processor and is controlled by a PC via USB.

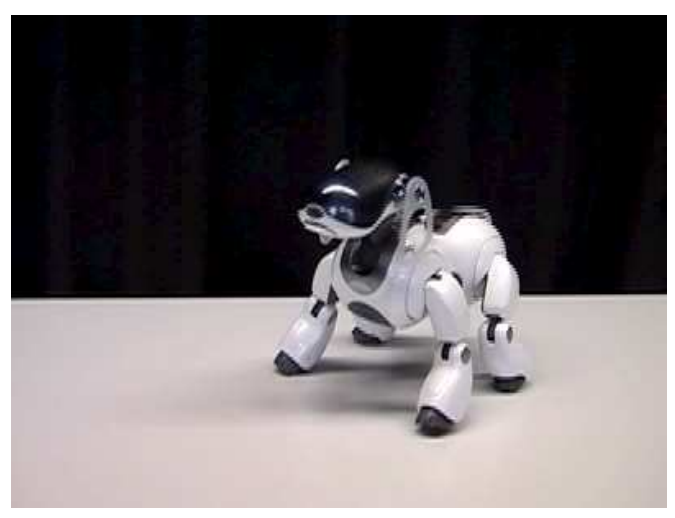

Fig. 2 Sony AIBO ERS-7

Even though the name suggests that the robot resembles a cat, its facial features are human-like. The iCat can create facial expressions by moving its eyebrows, eyelids, eyes, lips, and the head controlled by $13 \mathrm{RC}$ servos and 2 DC motors. We belief that, according to the categorization of Fong et al. (see II A [17]), the iCat is a caricatured robot. Both the robot's mouth and eyes are big and, thus, emphasized compared to a human face.

Next to the facial features, the robot has LEDs located in the feet and the ears to communicate its mode of operation (e.g. 
sleeping, awake, busy, or listening). The USB webcam in the nose of the iCat can be used for different computer vision tasks such as object and face recognition. Stereo microphones, a loudspeaker, and a soundcard placed in the feet of the robot are used for recording and playing sounds and speech. Thus, it is possible to use the platform for speech recognition tasks.

The iCat was primarily developed as a research platform and as an interface robot. As an interface the robot should be able to coordinate different electronic appliances like VCRs, or refrigerators in the intelligent home. Users can employ the Open Platform for Personal Robots (OPPR) software to program certain behaviours and applications. More details can be looked up in [41].

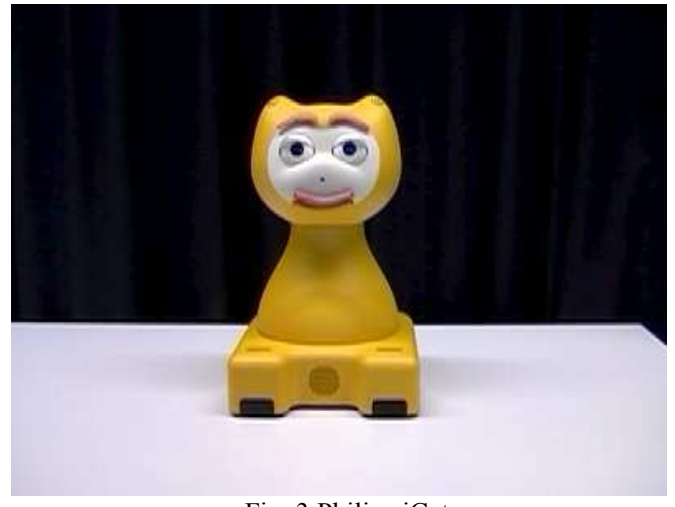

Fig. 3 Philips iCat

\section{BARTHOC (Bielefeld University)}

Fig. 6 gives an impression of the humanoid robot BARTHOC (Bielefeld Antropomorphic RoboT for HumanOriented Communication). This robot is designed by Bielefeld University in cooperation with Mabotic.

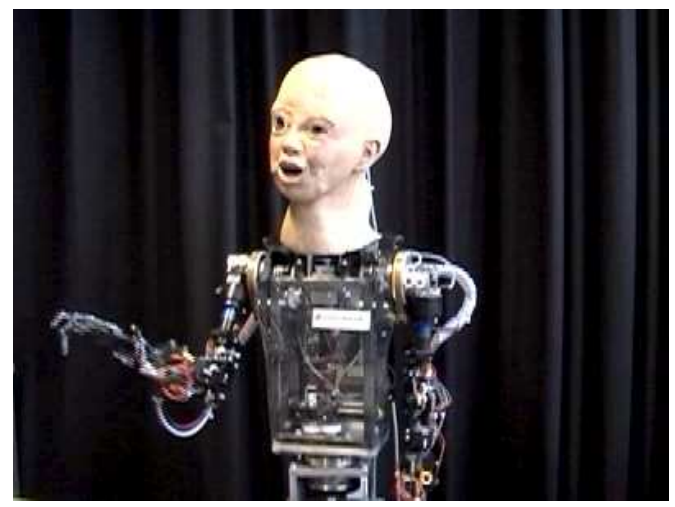

Fig. 4 BARTHOC ((Bielefeld Antropomorphic RoboT for Human-Oriented Communication)

BARTHOC consists of a mechatronic head with human-like features and two arms including hands. These components are mounted on a steel-frame backbone. Each arm has three joints similar to the human ones. The given degrees of freedom allow BARTHOC to perform human-like gestures. Actuators next to the upper lip and above the eyes simulate movements of lips and eyebrows which leads to basic human facial expressions. A camera is integrated in each eyeball for stereovision and microphones are currently placed on the shoulders. Additionally, a removable latex mask is available to equip the robot with different characters.

The robot is mainly used for research on human-like communication. One scenario the development of BARTHOC is based on is the receptionist scenario. In this scenario the robots is placed at the entrance of a building. It has to welcome people, to give general information, and to show the way to certain locations the visitor wants to got to by pointing and spoken instructions [42]. In [35] Hackel et al. describe BARTHOC in depth.

\section{BIRON (Bielefeld University)}

The robot BIRON (BIelefeld RObot companioN) (Fig. 7), developed at Bielefeld University, is based on an ActiveMedia $^{\text {TM }}$ Pioneer PeopleBot platform. BIRON is equipped with a pan-tilt colour camera at a height of $141 \mathrm{~cm}$

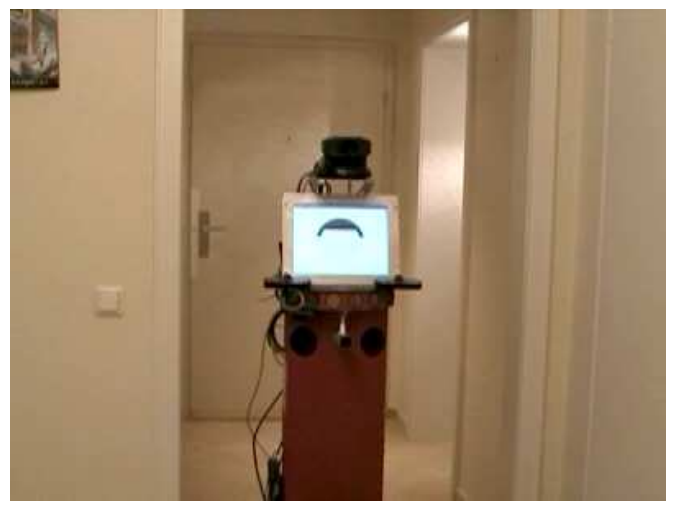

Fig. 5 BIRON (BIelefeld RObot companioN)

for acquiring images of the upper body part of humans interacting with the mobile robot. A pair of $A K G$ far-field microphones is located right below the touch screen display at a height of approximately $106 \mathrm{~cm}$. Therefore, BIRON has the capability to localize speakers. Speech processing is supported by a headset the human interaction partner wears. A SICK laser range finder mounted at a height of $30 \mathrm{~cm}$ facing front measures distances within a scene. Since BIRON has wheels, it can follow a person and move in a room. Additionally, it is able to track people and pay attention selectively to humans looking at it.

The robot is strongly embedded in the so-called home tour scenario. In this scenario the user orders a robot for the home which is delivered with all necessary sensors and capabilities but has to learn about its new environment to be able to fulfil tasks like getting things from a certain location. Thus, the user has to show the robot around the house and to teach it rooms and objects. Since most users are inexperienced the interaction with the system has to be designed as intuitive and natural as possible. Further information about BIRON is given in [43].

The robots described here were chosen for two reasons. Firstly, they have different appearances according to the categories of Fong et al. (see A, [17]). BIRON' design is functional, AIBO's zoomorphic, BARTHOC's anthropomorphic and iCat's caricatured. Secondly, we wanted 
to evaluate how our own robots developed for research compare to commercial products like iCat and AIBO.

\section{METHOD}

The research presented in this paper is based on an internet survey (see also [14]). This type of study was chosen for several reasons. First, it allows addressing people with different professional backgrounds, age and so forth. Thus, not only students or people related to a certain research field can be reached. Second, it can be conducted in a rather short time compared to live user trials. Third, the method supports the general idea of the study. People were asked to make a judgment based on the appearance of the robot in a video and a short description of its functionality. The internet provides a possibility to present this information without any further context and no technical problems with the robots could occur which would have influenced the subjects' answers. Therefore, we published the questionnaire in German on the website of an online laboratory. Some people were invited via private and professional mailing lists to participate. The mailing lists chosen were not connected to the robotics community.

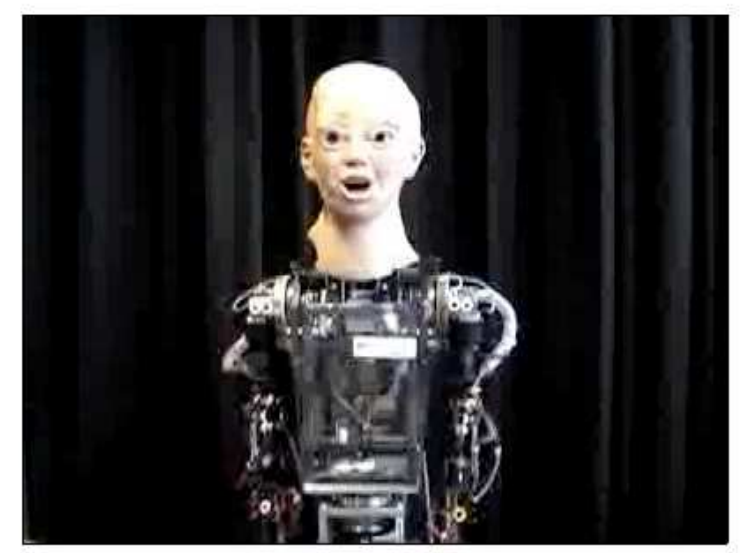

Das ist Barthoc. Barthoc kann sprechen und Sprache verstehen sowie Mimik und Gestik zeigen. Er kann Personen und Objekte in seiner Umgebung wahrnehmen und erkennen. Barthoc kann sich nicht fortbewegen.

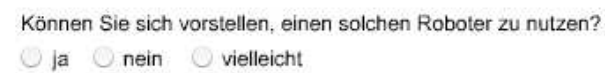

$\checkmark$ ja nein vielleicht

Welche sinnvollen Anwendungen kōnnen Sie sich prinzipiell für diesen Roboter vorstellen?

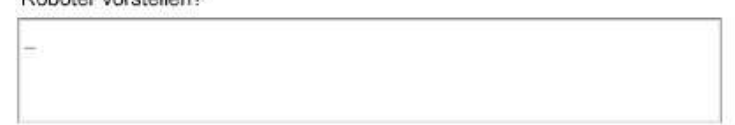

Welche Anwendungen für diesen Roboter würden Sie selbst nutzen?

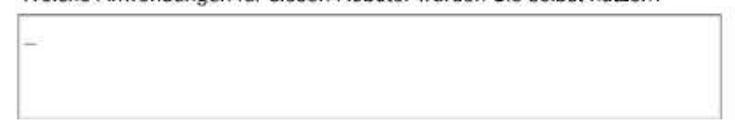

\section{weiter}

Fig. 6 Screenshot of questionnaire containing: description of BARTHOC; questions: Would you use this robot?; What useful applications could you imagine for this robot in principal?, Which applications of this robot would you use yourself?

The questionnaire commenced with general questions about the participants like age, profession, and knowledge about computers. The second part of the survey focused on the question which robots people knew. The participants were allowed to enter multiple robots, but they should not put down more than five. Moreover, they were asked to indicate where they knew the robots from (media, seen in real life, interacted with the robot, develops the robot, owns the robot). Thereafter, the four robots (AIBO, iCat, BIRON, BARTHOC) were introduced in random order (on one site each) (Fig. 2).

All participants received very basic information about the functionalities of the robots:

- $\quad$ AIBO: can speak and understand speech, it is able to walk and to recognize objects and people.

- iCat: can speak and understand speech, perceive and recognize people and objects in its environment, it can show facial expressions and reacts to touch, iCat is not mobile.

- BIRON: can speak and understand speech, recognize people and objects in its environment, it can remember the position of objects and people in a room, drive, and display information on its screen.

- BARTHOC: can speak and understand speech, it can show facial expressions and gestures, perceive and recognize people and objects in its environment, BARTHOC is not mobile.

In the descriptions we tried to use similar wording whenever possible in order to avoid different interpretations of the functionalities by the participants.

Next to the descriptions videos were displayed, which showed few movements of each system to give an impression of the robots' appearance. The movements shown in the video clips that lasted about 3 seconds were:

- $\quad$ AIBO: getting up on its feet and turning

- iCat: turning the head and blinking

- BIRON: driving towards the viewer

- BARTHOC: turning head and upper body and lifting the arm

We chose to display these very short videos with a neutral background to avoid any context like concrete tasks the robot could perform in a certain environment. In contrast to simple pictures the videos give the viewer a better sense of the embodiment of the robot. They were replayed as long as the subjects stayed on the given site of the questionnaire. Sound was turned off since the study mainly concentrated on visual appearance. Voices and background noise might have changed the perception of the robot significantly.

After reading the descriptions and watching the videos, subjects were asked to suggest applications for all four robots. Since the study was exploratory this question was an open question and the subjects were free to write down as many items as they could think of. The question was divided into a 
general and a personal one (What useful applications could you imagine for this robot in principal?, Which applications of this robot would you use yourself?). Moreover, participants had to indicate for each robot whether they could imagine using it at all. At the end of the questionnaire they were asked to decide (Fig. 3):

- Which of the robots shown would you like to own?

- Which robot is most enjoyable to interact with?

- Which robot is most likeable?

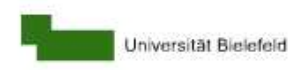

Welchen der vier Roboter würden Sie am liebsten besitzen?

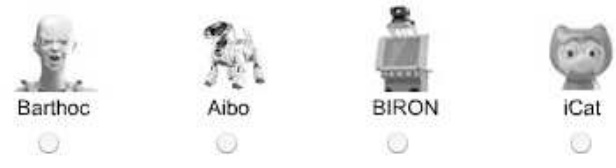

Versuchen Sie einzuschätzen, mit welchem der vier Roboter Ihnen die Interaktion am meisten Spaß machen würde.

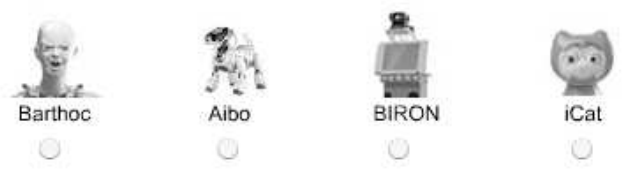

Welchen der vier Roboter finden Sie am sympathischsten?

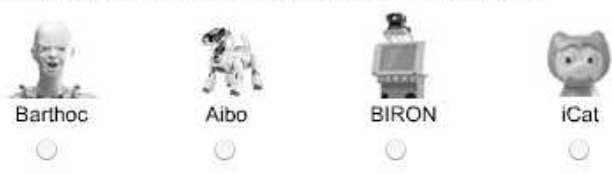

Bitte notieren Sie hier Ihre sonstigen Anmerkungen zum Experiment.
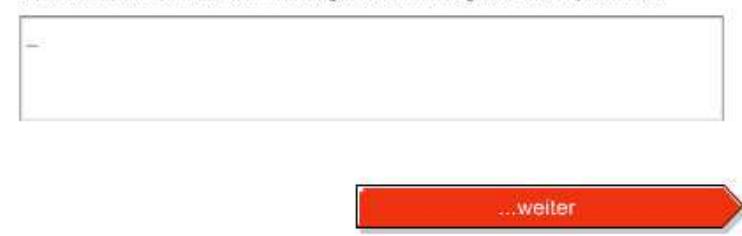

Fig. 7 Screenshot of questionnaire containing questions: Which of the robots shown would you like to own?, Which robot is most enjoyable to interact with?, Which robot is most likeable?

\section{V.RESULTS}

In [15] we have proposed four dimensions for the distinction between the applications named by the participants of the web survey: (1) public vs. private use, (2) intensity of interaction, (3) complexity of interaction model, (4) and functional vs. human-like appearance. In the centre of the paper at hand is an in-depth analysis of the first dimension (public vs. private use). This dimension describes to which degree the robot is suitable for public or domestic use. Starting point of the analysis is the matching hypothesis (see II B, [1]). It is assumed that if the appearance has to match the task of a robot, viewers will also suggest certain tasks for a robot mainly based on its appearance and also on its functionalities.
The analysis is based on the data from the web survey. Implications for the design of domestic robots will be identified. In the sense used in this paper the terms domestic and private are interchangeable.

Altogether, 127 people participated in the survey $(61 \%$ male, 39\% female). Most of the participants have German nationality (92\%), which is due to the fact that the questionnaire was published in German (0.8\% Swiss, $2.4 \%$ Austrian, $4.8 \%$ other). Their age ranged between 9 and 65 years (average $=27.4$ years). The educational background of the subjects was: $32.3 \%$ high school graduates; $55.9 \%$ university graduates; $9.5 \%$ doctoral degree; $2.3 \%$ other. $94 \%$ indicated to use computers both at home and in their job. They rated their own knowledge about computers fairly high (25\% expert, $67 \%$ advanced knowledge). This data shows that the sample was not representative for what we would call the general public. On the other hand, we suppose that people taking part in such a study are rather representative for the future user group of the robots. Nevertheless, the validity is restricted to Germany. Therefore, a further study is already set up in English. For the German survey presented here, participants needed an average of $11 \mathrm{~min} 21 \mathrm{sec}$.

As described above, in the second part of the questionnaire people were asked to write down names of robots they knew. Altogether 284 entries were made (mean=2.39, $\mathrm{SD}=1.73$ ). The robots mentioned most often were robots from TV and media (R2D2, C3PO etc.) (71), industrial robots (49), AIBO (23), service robots (21), and soccer robots (18). $71 \%$ indicated to know the robots from the media, $26 \%$ had seen a robot in real life, and only $9 \%$ had actually interacted with a robot before. Only four participants had used a domestic robot at home. All robots used at home were toy robots like Lego Mindstorms. Seven people had worked with a robot at a university.

As mentioned above, some participants had seen robots in real life. These were mainly industrial robots at work or at an exhibition. Some had seen AIBO at a soccer competition. Altogether, participants can be regarded as rather inexperienced in the field of robotics. One fact that has to be kept in mind though is that $18 \%$ knew AIBO, which is one of the robots shown in the study. The question if this has an effect on peoples' judgement of the robot will be addressed later in this section.

Altogether, people show a widespread rejection to using the robots (Table 1). The following comments taken from the questionnaires underline this (quotations were translated from German to English by the authors): "I don't want any robot at all!", "People or animals should not be replaced by robots.", "I don't think it's desirable to live together with a robot.", "I don't think that there are any useful applications for the robots shown here.", and "The robots scare me.". Participants made many more similar comments. As Kaplan [44] argues this is typical for the Western in contrast to the Japanese culture. One reason for rejecting robots might be that we still lack applications, which add some value to peoples' lives [19]. This explains why the willingness to use BIRON, the functionally designed robots, is highest (21.2\%). As will be seen later in 
this section, quite a few participants could imagine using this robot especially in a public context.

TABLE 1

WILLINGNESS TO USE THE ROBOTS (SAMPLE (N); YES, MAYBO, NO IN \%)

\begin{tabular}{lccccc}
\hline & AIBO & iCat & BARTHOC & BIRON & average \\
\hline $\mathrm{n}$ & 121 & 124 & 122 & 118 & \\
yes & 15.7 & 9.7 & 9.0 & $\mathbf{2 1 . 2}$ & 13.9 \\
maybe & 23.9 & 25.8 & 26.2 & 27.1 & 25.8 \\
no & 60.3 & 64.5 & 64.8 & 51.7 & 60.3 \\
\hline
\end{tabular}

TABLE 2

RATING (IN \%) OF THE QUESTIONS: (A) WHICH ROBOT WOULD YOU LIKE TO OWN? (B) WHICH ROBOT IS MOST ENJOYABLE? (C) WHICH ROBOT IS MOST LIKEABLE?; CORRELATION BETWEEN THE ANSWERS: ** $\mathrm{P}<.01$.; SAMPLE (N)

\begin{tabular}{lccccccc}
\hline & $\mathrm{n}$ & Aibo & iCat & Barthoc & Biron & (a) & (b) \\
\hline (a) & 111 & $\mathbf{4 4 . 1}$ & 17.1 & 5.4 & $\mathbf{3 3 . 3}$ & & \\
(b) & 114 & $\mathbf{5 7 . 0}$ & 16.7 & 9.7 & 16.7 & $.47^{* *}$ & \\
(c) & 110 & $\mathbf{4 9 . 1}$ & $\mathbf{3 9 . 1}$ & 2.7 & 9.1 & $.41^{* *}$ & $\begin{array}{c}.33 * \\
*\end{array}$ \\
\hline
\end{tabular}

Although the participants rejected using the robots in general, they preferred AIBO when asked which robot they would like to own, they find most enjoyable to interact with, and they regard as most likeable (Table 2). Answers to all three questions are strongly correlated as can be seen in Table 2. The preference for AIBO might be due to its appearance. As [45] found, people tend to find the robot cute and feel attracted to it. Moreover, many people knew AIBO beforehand and, thus, an acquaintance effect might also explain the preference.

It was further found that many participants refused BARTHOC. Only $2.7 \%$ of the participants stated that it was the most likeable of the robot. Also least subjects would like to own the robot (5.4\%) and it was found less enjoyable (9.4\%). This finding was supported by the fact that seven participants even wrote that they find BARTHOC scary. This can be explained with the uncanny valley theory (see II C). BARTHOC was the most human-like robot in the study. Anyhow, its appearance is far from imitating a human in a believable manner. This was also true for the movement displayed in the video which was rather choppy. As the uncanny valley theory states, robots that are supposed to look human-like but are as unperfected as BARTHOC is at the moment, cause a feeling of eeriness in the interlocutor. Also BARTHOC is perceived as being abnormal. Even though its proportions are similar to a human, its surface and movements are not. Since the uncanny valley theory describes problems caused by appearance and movements, it can be assumed that this is the reason for the bad rating of BARTHOC since the robot is not lagging behind the other robots regarding functionality.

\section{APPLICATION CATEGORIZATION}

As the central question of the survey, participants were asked to propose applications for AIBO, BIRON, BARTHOC, and iCat. Altogether 570 items were mentioned (see Table 3). Gender and age did not have a statistically significant influence on subjects' answers. As for the robots, there is a strong correlation between applications proposed for AIBO and $\mathrm{iCat}(\mathrm{r}=.77 ; \mathrm{p}<.01$ (two-tailed)). It can be assumed that this is due to appearance. As explained above, both have animal like features. Moreover, being consumer products both robots convey a similar degree of product-ness (see Section II B) which the other robots do not have.

For further analysis a method to classify the applications was developed. First, a content analysis was conducted. Three researchers classified the applications mentioned by the participants of the study independently into a free set of functional categories which should not overlap. The categories were than discussed and compared. On this basis a final set of application categories was developed. Second, based on these classifications the applications were grouped (Table 3). If one participant had written down two or more applications belonging to the same category the applications were counted as one. Every category was assigned to one of the higher-level environment-oriented groups domestic applications, public applications, or both domestic and public applications. In the focus of this paper are the domestic applications. However, to identify special requirements for these it will be necessary to contrast them with the other groups. Thus, firstly all categories are described and it is explained why certain applications are assigned to a certain group.

\section{A. Domestic Applications}

The first group consists of domestic applications. Within this group, Healthcare refers to robots used for therapy (e.g. autism therapy) and as support for sick or old people. This category also includes Caregiver robots that are used to watch old or sick people when nobody else is around. Companionship consists of all robots that keep company.

TABLE 3

APPLICATIONS PROPOSED FOR THE ROBOTS BY THE PARTICIPANTS SORTED BY CONTEXT OF USE

\begin{tabular}{lccccc}
\hline Applications & Aibo & iCat & Barthoc & Biron & total \\
\hline domestic: & & & & & \\
Healthcare / & $\mathbf{1 9}$ & $\mathbf{2 2}$ & 8 & 8 & 57 \\
Care giving & & & & & \\
Companionship & $\mathbf{8}$ & 3 & 2 & 2 & 15 \\
Entertainment & $\mathbf{1 0}$ & $\mathbf{8}$ & 2 & & 20 \\
Toy & $\mathbf{5 5}$ & $\mathbf{4 4}$ & 5 & 1 & $\mathbf{1 0 5}$ \\
Pet & $\mathbf{2 0}$ & 6 & & & 26 \\
Personal Ass. / & 7 & $\mathbf{1 4}$ & 3 & $\mathbf{3 0}$ & 54 \\
Interface & & & & & \\
both: & & & & & \\
Security & 23 & 17 & 5 & $\mathbf{3 2}$ & $\mathbf{7 7}$ \\
Teacher & 4 & $\mathbf{1 6}$ & 5 & 3 & 28 \\
Research & $\mathbf{1 1}$ & 1 & 4 & 2 & 18 \\
Transport & $\mathbf{1 4}$ & & 2 & 5 & 21 \\
(Fetch \& Carry) & & & & & \\
public: & & & & & \\
Business & 3 & 12 & $\mathbf{3 2}$ & 12 & 59 \\
Public Assistant & 6 & 13 & 27 & $\mathbf{4 4}$ & $\mathbf{9 0}$ \\
\hline total & 180 & 156 & 95 & 139 & $\mathbf{5 7 0}$ \\
\hline
\end{tabular}

According to the theory of anthropomorphism by Epley et 
al. [30] people anthropomorphize to a higher extent if they feel lonely, because for a human being there is a principle need for social connection. Loneliness elicits social pain, which hurts in the same way as physical pain [45]. Actually, e.g. elderly pet owners appear to be buffered from negative impact of stressful life events and visit a doctor less often compared to elderly people without pets [47]. Therefore, both Caregiver and Companionship are applications where robots might compensate the feeling of loneliness or social pain, respectively. This is what differentiates these categories from entertainment. The sole purpose of Entertainment robots is to entertain their users and to be a pastime. They are not built to have a psychological impact. The same is true for Toy robots which are mainly used for playing. Most robots already being sold for domestic usage belong to this category. Another application, which has been mentioned above, is Pet robots. It implies that the human shows responsibility for the robot. Pet robots are animal-like in appearance and function and might take the place of a real pet. Personal assistant or Interface describes robots used as butlers, organizers or interfaces. This category includes robots for cleaning and other household chores.

All applications described so far are domestic. We want to contrast them with the ones Christensen differentiates. As mentioned in Section II A [16], he names three groups of domestic applications (entertainment, assistance for elderly and handicapped, everyday tasks). We propose six categories, however, his groups are part of the categorization introduced in this paper. It is assumed that entertainment is part of the entertainment category established here; assistance for elderly and handicapped is similar to Healthcare / Care giving; and robots for everyday tasks are included in Personal Assistants / Interface. As a result, the categorization presented here includes three more categories (Companionship, Toy, Pet). One could argue that Companionship could be included in Healthcare or in Entertainment, Toy and Pet could also be incorporated in the Entertainment or in the Companionship category. Nevertheless, there are some major reasons not to do so. Mainly the differentiation is motivated by the fact that the categories bring about different capabilities and / or appearances the robot has to have. While Companionship is more than Entertainment because the state of the user has to be taken into account by a successful companion it is less than a Healthcare robot or a Caregiver whose function is broader since it has more responsibility for the well being of a person. Again there is a functional difference between a Toy and an Entertainment robot. Fong et al. also differentiate between these two groups (see II A, [17]). While a person usually actively makes up games for a toy, an entertainment robot is restricted to specific pre-programmed tasks. The difference between Pet and other categories is mainly due to the appearance of the robot. As discussed for AIBO, the appearance of the robot in this case obviously causes certain feelings in the user that are similar to feelings caused by real pets.

\section{B. Public and Domestic Applications}

The following applications can be used in both public and domestic environments. Security applications include robots used for surveillance, military tasks, exploration, tasks that are dangerous for humans (e.g. minesweeping), and for protection. Another category includes robots that teach certain tasks or abilities. The robots in this case are supplements to real teachers especially when learning languages. Research applications are useful to learn about robotics and human nature. Thus, these applications also serve to improve the robots themselves. Transport robots are useful for all kinds of fetch and carry tasks.

As noted above, these tasks can be both public and domestic. Even though the application does not allow for a concrete allocation, the appearance of the robots might in some cases. Whereas it is imaginable to use iCat as a teacher in a public as well as in a domestic environment, it is more plausible - because of the appearance of the robots - to use BIRON for security in public whereas AIBO might be used for the same task at home. The same is true for Fetch \& Carry tasks, one reason being that AIBO could easily be overseen and stepped on in public environments.

\section{Public Applications}

The last group consists of public applications. Within this group, a Business robot is either a receptionist, a sales robot, or a robot used for representation. Public assistants are guides (museum), information terminals or translators. All these robots are usually used for a short time at once and with other possible interaction partners being around.

Altogether, it cannot be reassured that the classification is exhaustive. There might be more applications that did not come up in the study especially since only four robots were tested. Anyhow, the categories help to explain the resulting differences between the robots and to compare them to scenarios proposed by the developers.

\section{APPLICATIONS FOR THE ROBOTS}

While the last section has focused on the categorization of all applications we now elaborate on the applications mentioned for the four robots. It was found that the applications named most often were Toy (105), Public Assistant (90), and Security (77) indicating that potential users have quite different and more diverse ideas on robot applications than robot developers. This is presumably due to the fact that participants who are not working in the field of robotics know less about feasibility of certain applications. Nevertheless, most applications mentioned were quite realistic from a robotics point of view or at least not completely out of reach. One reason is, presumably, that participants' were explicitly asked for "useful" applications.

AIBO was mainly seen as a domestic robot, especially as a Toy (55) and a Pet (20). This might have been influenced by the fact that many cheaper robotic dogs are available off-theshelf as toys. The applications mentioned are in line with the 
notion of "entertainment" used by Sony. Participants also suggested that the robot could be used for Healthcare / Care giving (19). This category also includes tasks like guide dog. The fact that people mention tasks very typical for dogs highlights that AIBO indeed has a zoomorphic appearance. It also explains that many subjects suggest Fetch \& Carry things (14) and Security (23) (guard dog) as applications for the robot.

As discussed above, in contrast to AIBO iCat is not a complete cat but only a torso. It has a rather caricatured appearance which explains that it was seen as a Pet by only few people (6). Part of the robot's caricatured appearance are its human-like facial features. These are presumably the reason that many participants suggested Teacher (16) (especially for languages) as an application for the robot. As explained in Section II B, Duffy states that human-like attributes are necessary for meaningful interaction. Meaningful interaction is undoubtedly especially important in teaching scenarios, which are very goal oriented. Thus, iCat with its facial features in fact seems suitable for this task. On the other hand iCat, like AIBO, most strongly evoked the impression of being a Toy (44). Apart from this, 14 people suggested Personal assistant as an application, half of these Interface, which is the scenario proposed by the developers. Surprisingly the participants did not indicate that they knew iCat in advance which would have implied that they had also been familiar with this scenario beforehand. The robot was also strongly linked with Healthcare / Care giving (22). In the applications described in this category, iCat in a broader sense is also an interface - not between a human and its electronic devices but between an old or sick person and other people that are not physically present.

Least applications were written down for BARTHOC. Most of the ones named are public (Business - 32, Public Assistant - 27). This is underlined by the following quotation (translation by the authors): "I would use the robot for the tasks mentioned above but only in the public. I would not want to have such a thing at home". However, the applications proposed are in line with the receptionist scenario the developers had in mind.

BIRON was judged quite differently. It was the only robot accepted for both public and private applications. On the one hand, people named applications like personal assistant (30), which supports the home tour scenario the researchers are mainly working on. On the other hand, participants strongly suggested using the robot for Security (32) and Public assistance (44). In fact, some pretty similar systems are already used as museum tour guides (e.g. [7],[8]) which might have influenced the subjects.

\section{VIII.APPLICATIONS PARTICIPANTS WOULD USE}

Next to general applications, participants were asked to name applications they would use themselves. These were categorized just as the ones above. 12 people wrote down applications they would use with BARTHOC, 28 with iCat, 38 with AIBO, and 36 with BIRON. Altogether, 122 applications were mentioned (12 BARTHOC, 30 iCat, 42 AIBO, 38
BIRON). Almost all people that would use the robot could only imagine using it for one certain task. Again, by far the least applications were mentioned for BARTHOC and most for AIBO.

Looking at Table 4, the results underline the findings described so far. Participants would use AIBO and iCat in a domestic environment, whereas BARTHOC was seen as a public robot. While some people would use domestic applications for BIRON the preference to employ this robot in public was strengthened. This might also be due to the functionality of the robot in connection with the appearance. While a public assistant e.g. an information terminal might not need an arm, a personal assistant built to carry things around would urgently need one. A statement by one user underlines this (translation by the authors): "I need a mobile robot with a gripper so that it can really do something". This supports the idea that even though appearance is a crucial factor in robot design also functionality and its representation in the appearance of the robot plays an important role.

TABLE 4

APPLICATIONS OF ROBOTS PARTICIPANTS WOULD USE THEMSELVES SORTED BY CONTEXT OF USE

\begin{tabular}{lccccc}
\hline Applications & Aibo & iCat & Barthoc & Biron & total \\
\hline domestic: & & & & & \\
Healthcare / & 1 & 1 & & 2 & 4 \\
Care giving & & & & & \\
Companionship & 1 & & & & 1 \\
Entertainment & 2 & 3 & & & 5 \\
Toy & $\mathbf{1 6}$ & $\mathbf{9}$ & 2 & & 27 \\
Pet & 1 & 1 & & & 2 \\
Personal Ass. / & 5 & 5 & 1 & $\mathbf{8}$ & 19 \\
Interface & & & & & \\
both: & & & & & \\
Security & $\mathbf{8}$ & 3 & 1 & 3 & 15 \\
Teacher & 1 & 3 & & 1 & 5 \\
Research & 2 & & & & 2 \\
Transport & $\mathbf{4}$ & & & & 4 \\
(Fetch \& Carry) & & & & & \\
public: & & & & & \\
Business & & & 1 & $\mathbf{5}$ & 6 \\
Public Assistant & 1 & 5 & $\mathbf{7}$ & $\mathbf{1 9}$ & 32 \\
\hline total & 42 & 30 & 12 & 38 & $\mathbf{1 2 2}$ \\
\hline
\end{tabular}

Table 4 also reveals that people only use applications if they have a need to do so (see [19], Section II A). Obviously only few have a need for Healthcare / Care giving, Companionship and so forth. This finding illustrates the fact that target groups for certain applications have to be identified and the design has to be based mainly on their needs.

\section{CONCLUSION}

Altogether, it can be concluded that almost half of the applications proposed are domestic applications according to the categorization presented here ( $49 \%$ of all applications; $48 \%$ of applications the participants would use themselves). Moreover, many applications especially the ones mentioned 
for AIBO that are included in the group "both" are also very likely to being used in private as long as the appearance of the respective robot is appropriate. These findings give quite some evidence on the importance that social domestic robots will gain in the future.

Moreover, they indicate that appearance in fact plays a crucial role in the perception of a robot and determines which applications are proposed. This should be kept in mind when designing a robot. In contrast to appearance the functionalities of the robot seem to be less important. They become crucial when something is missing for a certain scenario the participants would like to have (e.g. the gripper for BIRON in a domestic assistant scenario). One has to keep in mind, however, that these results are based on an internet survey. They might and probably will change in live human-robot interaction. However, appearance should be consciously designed to help users build a mental model of what a robot's application is.

The study presented here is only a first step to research perception of appearance and capabilities of robots. Certainly, more studies with different robots and methodologies have to follow. Nevertheless, including potential users in the process of finding new applications and designing social robots has proven to be a promising means of doing research in the field of social robotics.

\section{ACKNOWLEDGMENT}

The authors would like to thank Agnes Swadzba, Sven Wachsmuth and Katharina Rohlfing for their help and advice regarding the design and the realization of the study.

\section{REFERENCES}

[1] J. Goetz, S. Kiesler, and A. Powers, 'Matching robot appearance and behavior to tasks to improve human-robot cooperation', in Proceedings of the 12th IEEE Workshop on Robot and Human Interactive Communication (ROMAN 2003), San Francisco, CA, 2003, pp. 55-60.

[2] Lego Mindstorms, http://mindstorms.lego.com

[3] Ugobe Pleo, http://www.pleoworld.com/

[4] WowWee Robosapien, www.wowwee.com/robosapien/robo1/ robomain.html

[5] iRobot Roomba, http://irobot.com/

[6] Roboceptionist, http://www.ri.cmu.edu/projects/project_523.html

[7] I. R. Nourbakhsh, C. Kunz, and T. Willeke, The mobot museum robot installations: a five year experiment, in Proc. Intelligent Robots and Systems, 2003. (IROS 2003), vol.3, 2003, pp. 3636 - 3641.

[8] M. Bennewitz, F. Faber, D. Joho, M. Schreiber, and S. Behnke, Towards a Humanoid Museum Guide Robot that Interacts with Multiple Persons, in: Proceedings of the International Conference on Humanoid Robots (Humanoids), 2005.

[9] B. Duffy, 'The Social Robot', Ph.D. Thesis, Department of Computer Science, University College, Dublin, 2000.

[10] B. Duffy, 'Anthropomorphism and The Social Robot', Special Issue on Socially Interactive Robots, Robotics and Autonomous Systems 42 (3-4), 2003.

[11] C. Breazeal, A. Brooks, J. Gray, G. Hoffman, C. Kidd, H. Lee, J. Lieberman, A. Lockerd, and D. Chilongo, 'Tutelage and collaboration for humanoid robots', International Journal of Humanoid Robots, 1(2), 2004, pp. 315-348.

[12] C. Breazeal, 'Designing Sociable Robots'. Cambridge, MA, USA, MIT Press, 2002.

[13] C. Bartneck, J. Forlizzi, 'A Design-Centred Framework for Social Human-Robot Interaction', Proceedings of RO-MAN 2004, Kurashiki,
2004, pp. 591-594.

[14] M. Lohse, F. Hegel, A. Swadzba, K. Rohlfing, S. Wachsmuth, and B. Wrede, 'What can I do for you? Appearance and Applications of Robots' Proceedings of AISB'07, Artificial and Ambient Intelligence, Newcastle upon Tyne, 2007.

[15] F. Hegel, M. Lohse, A. Swadzba, K. Rohlfing, S. Wachsmuth, and B. Wrede, 'Applications of Social Robots: A User Study', in Proc. Int. Symposium on Robot and Human Interactive Communication (ROMAN), Jeju Island, Korea, 2007.

[16] H. I. Christensen, Intelligent home appliances. In Robotics Research, R. A. Jarvis and A. Zelinsky, Eds., no. 6 in Springer Tracts in Advanced Robotics (STAR). Springer Verlag, Heidelberg, Germany, January 2003, pp. 319-330.

[17] T. W. Fong, I. Nourbakhsh, and K. Dautenhahn, 'A Survey of Socially Interactive Robots: Concepts, Design, and Applications', Robotics and Autonomous Systems, 42(3-4), 2002, pp. $142-166$.

[18] S. Ljungblad and L. E. Holmquist, 'Designing robot applications for everyday environments', in sOc-EUSAI '05: Proceedings of the 2005 joint conference on Smart objects and ambient intelligence, New York, NY, USA, ACM Press, 2005, pp. 65-68.

[19] F. Kaplan, 'Everyday robotics: robots as everyday objects', in Proceedings of Soc-Eusai 2005, Grenoble, France, 2005, pp. 59 - 64.

[20] C. Bartneck, 'Interacting with and embodied emotional Character'. Proceedings of the DPPI2003 Conference, Pittsburgh, 2003, pp. 55-60.

[21] Y. Jung and K. M. Lee (2004). 'Effects of physical embodiment on social presence of social robots'. Proceedings of Presence, 2004, pp. 8087.

[22] L. Sullivan, The Tall Office Building Artistically Considered, in Kindergarten Chats (revised 1918) and Other Writings, edited by I. Athey, New York: Wittenborn, Schultz, 1947, pp. 202-13.

[23] M. Blow, K. Dautenhahn, A. Appleby, C. L. Nehaniv, and D. C. Lee, 'Perception of Robot Smiles and Dimensions for Human-Robot Interaction Design' 15th IEEE International Symposium on Robot and Human Interactive Communication (RO-MAN 06), pages 469-474, Hatfield, UK, (2006).

[24] J. Donat,. 'Mediated Faces', in M. Beynon, C. L. Nehaniv, K. Dautenhahn (Eds.). Cognitive Technology: Instruments of Mind. Proceedings of the 4th International Conference, CI 2001, Warwick, UK, 2001.

[25] R. Gockley, J. Forlizzi, and R. Simmons, 'Interactions with a moody robot', in HRI '06: Proceeding of the 1st ACM SIGCHI/SIGART conference on human-robot interaction, New York, NY, USA, ACM Press, 2006, pp. 186-193.

[26] A. Bruce, I. Nourbakhsh, and R. Simmons, 'The role of expressiveness and attention in human-robot interaction', in Proc. AAAI Fall Symp. Emotional and Intel. II: The Tangled Knot of Soc. Cognition, 2001.

[27] F. Hegel, T. Spexard, T. Vogt, G. Horstmann, and B. Wrede, 'Playing a different imitation game: Interaction with an Empathic Android Robot', in Proc. 2006 IEEE-RAS International Conference on Humanoid Robots (Humanoids06), IEEE, 2006, pp. 56-61.

[28] C. F. DiSalvo, F. Gemperle, J. Forlizzi, and S. Kiesler, 'All robots are not created equal: the design and perception of humanoid robot heads', in DIS '02: Proceedings of the conference on Designing interactive systems, pp. 321-326, New York, NY, USA, ACM Press, 2002.

[29] F. Hegel, S. Krach, T. Kircher, B. Wrede, and G. Sagerer, 'Theory of Mind (ToM) on Robots: A Neuroimaging Study', Proceedings of Human-Robot Interaction (HRI 2008), Amsterdam, Netherlands, 2008.

[30] N. Epley, A. Waytz, and J. T. Cacioppo, 'On Seeing Human: A threefactor theory of anthropomorphism', Psychological Review, 114, 2007, pp. 864-886.

[31] H. v. Foerster, 'Wissen und Gewissen. Versuch einer Brücke', Frankfurt, 1997.

[32] S. E. Guthrie, 'Anthropomorphism: A Definition and a Theory', in: R. W. Mitchell, N. S. Thompson, and H. L. Miles, 'Anthropomorphism, anecdotes, and animals'. Albany: State University of New York Press, 1997.

[33] C. K. Morewedge, J. Preston, and D. M. Wegner, 'Timescale is in the attribution of mind', Journal of Personality and Social Psychology, 93, 2007, pp. 1-11.

[34] D. Sakamoto, T. Kanda, T. Ono, H. Ishiguro, and N. Hagita, 'Android as a Telecommunication medium with Human Like Presence'. ACM/IEEE 2nd Annual Conference on Human-Robot Interaction (HRI2007), Arlington, Virginia, USA, 2007, pp. 193-200. 
[35] M. Hackel, M. Schwope, J. Fritsch, B. Wrede, and G. Sagerer, 'Designing a sociable humanoid robot for interdisciplinary research', Advanced Robotics, 20(11), 2006, pp. 1219-1235.

[36] K. F. MacDorman and H. Ishiguro, 'The uncanny advantage of using androids in social and cognitive science research', Interaction Studies, 7(3), 2006, pp. 297-337.

[37] M. Mori, 'Bukimi no tani'. (Translation by K. F. MacDorman and T. Minato, http://www.androidscience.com/theuncannyvalley/ proceedings2005/uncannyvalley.html). Energy, 7(4), 1970, pp. 33-35, (Originally in Japanese).

[38] K. F. MacDorman, 'Subjective ratings of robot video clips for human likeness, familiarity, and eeriness: An exploration of the uncanny valley', ICCS/CogSci-2006 Long Symposium: Toward Social Mechanisms of Android Science. July 26, 2005. Vancouver, Canada, 2005.

[39] J. Seyama and R. S. Nagayama, The uncanny valley: Effect of realism on the impression of artificial human faces, Presence: Teleoperators and Virtual Environments, 16(4), 2007, pp. 337-351.

[40] Sony AIBO website. http://support.sony-europe.com/aibo/

[41] Philips Research, iCat research community, http:// www.hitechprojects.com/icat/
[42] N. Beuter, "Gestenbasierte Positionsreferenzierung für die multimodale Interaktion mit einem anthropomorphen Robotersystem", diploma thesis, Faculty of Technology: Bielefeld University, 2007.

[43] A. Haasch, S. Hohenner, S. Hüwel, M. Kleinehagenbrock, S. Lang, I. Toptsis, G. A. Fink, J. Fritsch, B. Wrede, and G. Sagerer, 'BIRON - The Bielefeld Robot Companion', in Proc. Int. Workshop on Advances in Service Robotics, Eds. E. Prassler, G. Lawitzky, P. Fiorini, and M. Hägele, Stuttgart, Germany, Fraunhofer IRB Verlag. 2004, pp. 27-32.

[44] F. Kaplan, 'Who is afraid of the humanoid? Investigating cultural differences in the acceptance of robots', International journal of humanoid robotics, 1(3), 2004, pp. 465-480.

[45] C. Bartneck, J. Reichenbach, and J. Carpenter, Use of Praise and Punishment in Human-Robot Collaborative Teams, Proceedings of the RO-MAN 2006 - The 15th IEEE International Symposium on Robot and Human Interactive Communication, Hatfield, 2006, pp. 177-182.

[46] G. MacDonald and M. R. Leary, Why does social exclusion hurt? The relationship between social and physical pain, Psychological Bulletin, 131, 2005, pp. 202-223.

[47] J. M. Siegel, Stressful life events and use of physician services among the elderly: The moderating role of pet ownership, Journal of Personality and Social Psychology, 58, 1990, pp. 1081-1086. 